\title{
SEGURANÇA ALIMENTAR E NUTRICIONAL DE BENEFICIÁRIOS DO BOLSA FAMÍLIA DE UM MUNICÍPIO DA REGIÃO NOROESTE DO PARANÁ
}

Paula Montanhini Favetta1, Dirlene Pereira de Lima², Grazielli de Fátima Serenini ${ }^{3}$ Diego Sandro Bedendo Barbosa ${ }^{4}$, Luciana Kazue Otutumi ${ }^{5}$, Ricardo de Melo Germano $^{6}$

1 Nutricionista. Mestranda do Programa de Pós-Graduação em Ciência Animal com

Ênfase em Produtos Bioativos - UNIPAR (favettap@outlook.com)

2 Nutricionista. Mestre em Ciência Animal pela Universidade Paranaense - UNIPAR.

Docente do Curso de Nutrição - UNIPAR

3 Bióloga. Mestranda do Programa de Pós-Graduação em Ciência Animal com

Ênfase em Produtos Bioativos - UNIPAR

4 Educador Físico. Acadêmico do curso de Nutrição - UNIPAR

5 Médica Veterinária. Pós-Doutora em Microbiologia pela Universidade de São Paulo. Docente no Programa de Pós-Graduação em Ciência Animal com Ênfase em Produtos Bioativos - UNIPAR

6 Doutor em Biologia das Interações Orgânicas pela Universidade Estadual de Maringá. Docente no Programa de Pós-Graduação em Ciência Animal com Ênfase em Produtos Bioativos - UNIPAR e do Mestrado Profissional em Plantas Medicinais

e Fitoterápicos na Atenção Básica - UNIPAR

\section{Recebido em: 06/04/2019 - Aprovado em: 10/06/2019 - Publicado em: 30/06/2019} DOI: 10.18677/EnciBio_2019A164

\begin{abstract}
RESUMO
Com o presente estudo pretendeu-se obter uma visão geral do nível de Segurança Alimentar e Nutricional (SAN). A SAN é caracterizada pela garantia do acesso regular e permanente a alimentos com qualidade e quantidade suficientes, com educação nutricional, econômica, ambiental e de forma sustentável. Os participantes do presente estudo eram do Programa Bolsa Família do município de UmuaramaPR, atendidos no Centro de Referência e Assistência Social - CRAS II. Afim de identificar os efeitos da SAN que o Programa Bolsa Família tem na vida dos beneficiários, em comparativo aos que não usufruem do programa. Por intermédio da Escala Brasileira de Insegurança Alimentar - EBIA e por um Questionário de Frequência Alimentar - QFA, foi possível identificar as escolhas alimentares da população estudada e a classificação dos níveis da Segurança Alimentar. Nas condições em que foi conduzido o estudo, os resultados não comprovaram a eficácia do programa Bolsa Família na vida dos beneficiários, uma vez que não houve diferença significativa entre os grupos, além disso, as escolhas alimentares observadas por meio do QFA, apontaram elevado consumo para alimentos de baixa qualidade nutricional nos participantes classificados em SAN. Os resultados também demonstraram maior índice no consumo de alimentos de baixo valor nutricional para participantes classificados em Insegurança Alimentar Leve, permitindo concluir que são necessárias ações que promovam a educação nutricional, bem como, melhorias nas escolhas alimentares, de modo que ocorra melhora no estado e nas condições nutricionais desta população.
\end{abstract}

PALAVRAS-CHAVE: Insegurança alimentar. Nutrição. Programa Bolsa Família. 


\title{
FOOD AND NUTRITIONAL SAFETY OF BENEFICIARIES OF THE FAMILY BAG OF A MUNICIPALITY OF THE NORTHWEST REGION OF PARANÁ
}

\begin{abstract}
The present study aimed to obtain an overview of the level of Food and Nutrition Security (SAN). The SAN is characterized by the guarantee of regular and permanent access to foods of sufficient quality and quantity, with nutritional, economic, environmental and sustainable education. The participants of the present study were from the Bolsa Família Program of the municipality of Umuarama-PR, attended at the Reference and Social Assistance Center - CRAS II. In order to identify the effects of the SAN that the Bolsa Família Program has on the lives of the beneficiaries, in comparison to those who do not benefit from the program. Through the Brazilian Food Insecurity Scale - EBIA and through a Food Frequency Questionnaire - FFQ, it was possible to identify the food choices of the studied population and the classification of Food Safety levels. In the conditions under which the study was conducted, the results did not prove the effectiveness of the Bolsa Familia program in the life of the beneficiaries, since there was no significant difference between the groups, in addition, the food choices observed through the FFQ, indicated high consumption for foods of low nutritional quality in the participants classified in SAN. The results also showed a higher index in the consumption of foods of low nutritional value for participants classified in Light Food Insecurity, allowing to conclude that actions are necessary that promote nutritional education, as well as improvements in food choices, so that there is an improvement in the state and nutritional conditions of this population.
\end{abstract}

KEY WORDS: Food insecurity. Family Grant Program. Nutrition.

\section{INTRODUÇ̃̃O}

A Segurança Alimentar e Nutricional (SAN) é um direito humano que visa a alimentação acessível, de qualidade, em quantidade suficiente e de modo constante e permanente, evidenciando-se práticas alimentares que promovam a saúde sem comprometer o acesso a outras necessidades essenciais, além disso, é um direito brasileiro que deve, principalmente, respeitar as peculiaridades e características culturais de cada lugar (VASCONCELLOS; MOURA, 2018).

A insegurança alimentar (IA) pode ser caracterizada desde a fome por privação, incerteza e limitação do consumo de alimentos e produtos de qualidade, onde muitas vezes as pessoas optam por produtos de qualidade inferior que prejudicam a saúde, até preços abusivos e padrões alimentares impostos pela sociedade que não levam em consideração a diversidade cultural (SILVA et al., 2018).

Uma das principais causas da falta de SAN, se dá pela desigualdade na renda de muitos brasileiros, dificultando a obtenção de alimentos de forma regular, variada e permanente, apesar de ser um direito garantido pela legislação brasileira e os direitos humanos, que asseguram o adequado acesso à alimentação e nutrição de forma quantitativa e qualitativa (FARIA; DIAS, 2016).

Nesse contexto, foram criados Programas de Transferência Condicional de Renda voltadas a atender às necessidades da população carente, desde a educação até a alimentação, sendo que um destes é o Programa Bolsa Família (PBF), composto por famílias com menores rendas e consequentemente maior situação de IA (PINTO et al., 2017).

O PBF, que está em vigor desde 2004, tem como finalidade abrandar as diferenças sociais, realizando a transferência de renda direta e condicionada, com 
intenção de diminuir a pobreza e a fome, promovendo-se a segurança alimentar (COELHO; MELO, 2017)

De acordo com Ferreira et al. (2014), para avaliarem a IA dos indivíduos, família ou população, alguns métodos têm sido utilizados e testados em diversas entidades, buscando-se contemplar as diferentes dimensões da SAN: disponibilidade, acesso, utilização e estabilidade do alimento. No Brasil, a Escala Brasileira de Insegurança Alimentar (EBIA) é o método utilizado para avaliar a percepção da IA, acesso e disponibilidade aos alimentos, em ambientes populacionais, inquirindo-se o responsável pelo alimento no domicílio a uma série de questões subjetivas e classificando as famílias em estado de segurança alimentar ou insegurança alimentar, em diferentes níveis.

A EBIA é largamente utilizada no país devido ao baixo custo e sua consistência interna e externa, porém, pode ser aprimorada, com a inclusão de outros indicadores conforme a necessidade de sua aplicação local (FERREIRA et al., 2014). Essa escala foi proposta e validada para o Brasil por Segall-Corrêa et al. (2003), tendo sido reconhecida como indicador sensível para detectar famílias em risco de insegurança alimentar. A adaptação e a validação desse instrumento partiram da escala desenvolvida pelo United States Department of Agriculture (USDA), e aplicada em inquéritos populacionais do país desde 1995, bem como em outros países desenvolvidos e subdesenvolvidos (YUYAMA et al., 2008).

Constata-se também que a SAN nunca ocupou tamanho espaço na agenda política no Brasil, visto que diversos setores governamentais e não governamentais investem no desenvolvimento de ações que busquem a garantia do direito à segurança alimentar e nutricional à população, buscando ações mais efetivas, a fim de dar-lhes sustentabilidade (FERREIRA et al., 2014).

Visto a realidade local, o objetivo do presente estudo foi de conhecer o nível de SAN de participantes e não participantes do Programa Bolsa Família do município de Umuarama-PR atendidos no Centro de Referência à Assistência Social - CRAS II afim de identificar os efeitos que a SAN e o PBF impelem aos que nele se inserem, identificando o atendimento ou não das necessidades dessa população.

\section{MATERIAL E MÉTODOS}

Todos os procedimentos metodológicos adotados foram aprovados pelo Comitê de Ética em Pesquisa com Seres Humanos da Universidade Paranaense UNIPAR sob o número do CAAE: 70435317.1.0000.0109. Todas as informações sobre a pesquisa e uso de dados foram esclarecidas a todos os participantes, sendo que a participação somente foi efetivada após leitura e assinatura do Termo de Consentimento Livre e Esclarecido - TCLE.

A população estudada é amparada pelo Centro de Referência da Assistência Social (CRAS II), na cidade de Umuarama, PR, estruturada para atender 59 bairros e, desses, 14 apresentam maior vulnerabilidade social, sendo que as famílias atendidas, têm como perfil a chefia de mulheres, apesar de a procura dos serviços do CRAS II por homens ter crescido em consequência do momento econômico de dificuldade vivenciada pelo país.

O CRAS II atende 600 usuários do PBF, com a mesma realidade socioeconômica, destes obteve-se uma amostra de $11,66 \%$ dividida igualmente em dois grupos. O percentual da amostra foi estabelecido com referência ao número total de pessoas atendidas, o qual representa um resultado significativo, totalizando 35 voluntários para cada grupo, beneficiários e não beneficiários do PBF, com o propósito de compará-los. Os questionários foram aplicados no CRAS II, nos 
períodos matutinos e vespertinos ao longo de 10 dias e também em reuniões realizadas pela assistente social.

Com o intuito de caracterizar a população a ser estudada, foram consideradas variáveis: socioeconômicas, disponibilidade domiciliar de alimentos, situação da segurança alimentar e da SAN. Foram aplicados dois questionários: Escala Brasileira de Insegurança Alimentar (EBIA) e Questionário de Frequência Alimentar (QFA) utilizando o marcador de consumo alimentar adaptado do SISVAN - Sistema de Vigilância Alimentar e Nutricional (BRASIL, 2008) afim de identificar as escolhas e hábitos alimentares desses grupos, baseados em alimentos de acordo com a realidade da amostra, e com alta e baixa qualidade nutricional.

O consumo alimentar foi classificado de acordo com os tipos de alimentos e a quantidade em dias de consumo. Para os participantes da pesquisa que consumiam feijão, frutas, verduras, legumes, leites e iogurtes diariamente ou mais de três vezes na semana foram classificados em alto consumo e para os que consumiam menos de três vezes na semana estes alimentos, foram classificados em baixo consumo. $O$ mesmo ocorre para os alimentos altamente calóricos, quando ocorre o consumo de alimentos fritos e guloseimas como embutidos, refrigerante, doces, bolachas e biscoitos, por mais de três vezes na semana ou diariamente, foram classificados em alto consumo e quando consumidos até três vezes na semana, baixo consumo.

Cada resposta afirmativa na EBIA representou um ponto, o que mostra que a somatória desses pontos avalia a insuficiência alimentar, com pontuações diferentes para domicílios com pelo menos um morador com menos de 18 anos de idade, conforme apresentado nos Quadros 1 e 2.

Quando não há nenhuma resposta positiva, a família é classificada em situação de segurança alimentar e a classificação da insegurança alimentar em diferentes gradientes corresponde a patamares diferenciados da soma dos pontos obtidos no questionário.

QUADRO 1 - Pontuação para classificação dos domicílios com pelo menos um morador menor de 18 anos de idade.

\begin{tabular}{|c|c|}
\hline Pontuação & Categorias \\
\hline 0 pontos & Segurança Alimentar \\
\hline 1 a 5 pontos & Insegurança Alimentar Leve \\
\hline 6 a 10 pontos & Insegurança Alimentar Moderada \\
\hline 11 a 15 pontos & Insegurança Alimentar Grave \\
\hline
\end{tabular}

Fonte: PNAD, 2004 (IBGE, 2006)

QUADRO 2 - Pontuação para classificação dos domicílios com somente moradores acima de 18 anos de idade.

\begin{tabular}{|c|c|}
\hline Pontuação & Categorias \\
\hline 0 pontos & Segurança Alimentar \\
\hline 1 a 3 pontos & Insegurança Alimentar Leve \\
\hline 4 a 6 pontos & Insegurança Alimentar Moderada \\
\hline 7 a 9 pontos & Insegurança Alimentar Grave \\
\hline
\end{tabular}

Fonte: PNAD, 2009 (IBGE, 2010) 
QUADRO 3 - Descrição da situação de segurança alimentar, com o significado da situação encontrada no domicílio segundo os níveis de severidade de Insegurança Alimentar e Nutricional.

\begin{tabular}{|c|l|}
\hline $\begin{array}{c}\text { Segurança Alimentar } \\
\text { (SA) }\end{array}$ & $\begin{array}{l}\text { O domicílio tem acesso regular e permanente a alimentos } \\
\text { de qualidade, em quantidade suficiente, sem comprometer o } \\
\text { acesso a outras necessidades essenciais; }\end{array}$ \\
\hline $\begin{array}{c}\text { Insegurança Alimentar Leve } \\
\text { (IAL) }\end{array}$ & $\begin{array}{l}\text { Preocupação ou incerteza quanto ao acesso aos alimentos no } \\
\text { futuro; qualidade inadequada dos alimentos resultante de } \\
\text { estratégias que visam não comprometer a quantidade de } \\
\text { alimentos; }\end{array}$ \\
\hline $\begin{array}{c}\text { Insegurança Alimentar } \\
\text { Moderada } \\
\text { (IAM) }\end{array}$ & $\begin{array}{l}\text { Redução quantitativa de alimentos entre os adultos e/ou ruptura } \\
\text { nos padrões de Alimentação resultante da falta de } \\
\text { alimentos entre os adultos; }\end{array}$ \\
\hline $\begin{array}{c}\text { Insegurança Alimentar Grave } \\
\text { (IAG) }\end{array}$ & $\begin{array}{l}\text { Redução quantitativa de alimentos entre as crianças e/ou ruptura } \\
\text { nos padrões de alimentação resultante da falta de alimentos entre } \\
\text { as crianças; fome (quando alguém fica o dia inteiro sem comer por } \\
\text { falta de dinheiro para comprar alimentos). }\end{array}$ \\
\hline
\end{tabular}

Após preenchimento dos questionários, as somatórias de pontos foram realizadas e os resultados foram tabulados para realização da análise estatística. $\mathrm{O}$ programa utilizado foi o IBM SPSS v. 21.0. Para verificar a associação entre a classificação em relação à Insegurança Alimentar e a participação no Programa Bolsa Família foi utilizado o teste Exato de Fisher ao nível de $5 \%$ de significância, sendo realizadas comparações dois a dois. Além disso, foram elaboradas tabelas e gráficos a partir do uso da ferramenta Tabela de referência cruzada, relacionados aos tipos de alimentos consumidos (salgados fritos, embutidos, bolachas e biscoitos, doces e refrigerantes) os quais foram classificados como alto ou baixo consumo e a situação da SAN.

\section{A classificação da SAN}

\section{RESULTADOS E DISCUSSÃO}

No presente estudo, foram avaliadas 70 pessoas, das quais 35 eram beneficiárias do PBF e outras 35 não beneficiárias. Analisando os resultados dentro dos grupos de SAN, entre os integrantes do PBF, 60,0\% apresentaram situação de insegurança alimentar leve, 52,6\% demonstraram insegurança alimentar moderada, $50,0 \%$ com insegurança alimentar grave e somente $35,5 \%$ estão em situação de segurança alimentar. Em contrapartida, $64,5 \%$ dos não beneficiários encontram-se em situação de segurança alimentar (Tabela 1). No entanto, houve diferença significativa quando comparando o grupo que recebe assistência do programa e o grupo que não recebe.

TABELA 1: Comparativo entre os percentuais dos níveis de Segurança Alimentar e Nutricional dos usuários participantes e não participantes do Programa Bolsa Família.

\section{Situação de SAN \\ n Participantes do bolsa família}

\begin{tabular}{llc}
\hline Segurança alimentar & 17 & $6(35,5 \%)$ \\
Insegurança alimentar leve & 20 & $12(60,0 \%)$ \\
Insegurança alimentar moderada & 19 & $10(52,6 \%)$ \\
Insegurança alimentar grave & 14 & $7(50,0 \%)$
\end{tabular}

Todas as comparações não foram significativas pelo Teste Exato de Fisher $(P>0,05)$.

$\mathrm{n}=$ número de entrevistados 
Os resultados encontrados demonstram que a ascensão ao acesso de alimentos não é acompanhada pela qualidade alimentar, uma vez que as escolhas alimentares identificadas entre os dois grupos pelo QFA indicam consumo para alimentos de baixo valor nutricional tanto para os que estão em segurança alimentar quanto os que foram classificados em diferentes níveis de insegurança alimentar (Tabela 2).

TABELA 2: Escolhas alimentares obtidas de ambos os grupos pelo Questionário de Frequência Alimentar, para alimentos de baixo valor nutricional.

\begin{tabular}{|c|c|c|c|c|c|}
\hline \multirow[t]{2}{*}{ Alimentos } & \multirow[t]{2}{*}{ Condição } & \multirow{2}{*}{$\begin{array}{l}\text { Segurança } \\
\text { alimentar }\end{array}$} & \multicolumn{3}{|c|}{ Insegurança alimentar } \\
\hline & & & Leve & Moderada & Grave \\
\hline Salgados & Alto & $1 / 5(20,0 \%)$ & $2 / 5(40,0 \%)$ & $1 / 5(20,0 \%)$ & $1 / 5(20,0 \%)$ \\
\hline fritos & $\begin{array}{l}\text { consumo } \\
\text { Baixo } \\
\text { consumo }\end{array}$ & $\begin{array}{c}16 / 65 \\
(24,62 \%)\end{array}$ & $\begin{array}{c}18 / 65 \\
(27,69 \%)\end{array}$ & $\begin{array}{c}18 / 65 \\
(27,69 \%)\end{array}$ & $13 / 65(20,0 \%)$ \\
\hline Embutidos & $\begin{array}{l}\text { Alto } \\
\text { consumo } \\
\text { Baixo } \\
\text { consumo }\end{array}$ & $\begin{array}{c}3 / 5(60,0 \%) \\
14 / 65 \\
(21,54 \%)\end{array}$ & $\begin{array}{c}0 / 5(0 \%) \\
20 / 65 \\
(30,77 \%)\end{array}$ & $\begin{array}{c}2 / 5(40 \%) \\
17 / 65 \\
(26,15 \%)\end{array}$ & $\begin{array}{c}0 / 5(0 \%) \\
14 / 65(21,54 \%)\end{array}$ \\
\hline $\begin{array}{l}\text { Bolachas e } \\
\text { biscoitos }\end{array}$ & $\begin{array}{l}\text { Alto } \\
\text { consumo } \\
\text { Baixo } \\
\text { consumo }\end{array}$ & $\begin{array}{c}4 / 16 \\
(25,0 \%) \\
13 / 54 \\
(24,07 \%)\end{array}$ & $\begin{array}{c}6 / 16(37,5 \%) \\
14 / 54 \\
(25,93 \%)\end{array}$ & $\begin{array}{c}3 / 16(18,75 \%) \\
16 / 54 \\
(29,63 \%)\end{array}$ & $\begin{array}{c}3 / 16(18,75 \%) \\
11 / 54(20,37 \%)\end{array}$ \\
\hline Doces & $\begin{array}{l}\text { Alto } \\
\text { consumo } \\
\text { Baixo } \\
\text { consumo }\end{array}$ & $\begin{array}{c}4 / 14 \\
(28,6 \%) \\
13 / 56 \\
(23,2 \%)\end{array}$ & $\begin{array}{c}5 / 14(35,7 \%) \\
15 / 56(26,8 \%)\end{array}$ & $\begin{array}{c}2 / 14(14,3 \%) \\
17 / 56(30,4 \%)\end{array}$ & $\begin{array}{c}3 / 14(21,4 \%) \\
11 / 56(19,6 \%)\end{array}$ \\
\hline Refrigerante & $\begin{array}{l}\text { Alto } \\
\text { consumo } \\
\text { Baixo } \\
\text { consumo }\end{array}$ & $\begin{array}{c}3 / 7 \\
(42,86 \%) \\
14 / 63 \\
(22,22 \%)\end{array}$ & $\begin{array}{c}0 / 7(0 \%) \\
20 / 63 \\
(31,75 \%)\end{array}$ & $\begin{array}{c}3 / 7(42,86 \%) \\
16 / 63(25,4 \%)\end{array}$ & $\begin{array}{c}1 / 7(14,29 \%) \\
13 / 63(20,63 \%)\end{array}$ \\
\hline
\end{tabular}

Percentual calculado com base no total de participantes classificados dentro da categoria alto ou baixo consumo.

O estudo também evidencia que, estar em SAN não significa ter uma alimentação em quantidade e qualidade, onde, mesmo os classificados em SAN apresentam alto consumo de refrigerantes e embutidos (Figura A). Apesar do baixo consumo de embutidos e refrigerante, o grupo classificado em IAL (37,5\%), demonstrou grande percentual de consumo para doces, bolachas e biscoitos e salgados fritos (40\%) (Figura B). Para os alimentos considerados de alto valor nutricional, o consumo observado pelo grupo classificado em IAL, foi de maior porcentagem para leites e iogurtes, frutas, legumes cozidos e salada crua (Figura C e Tabela 3). 

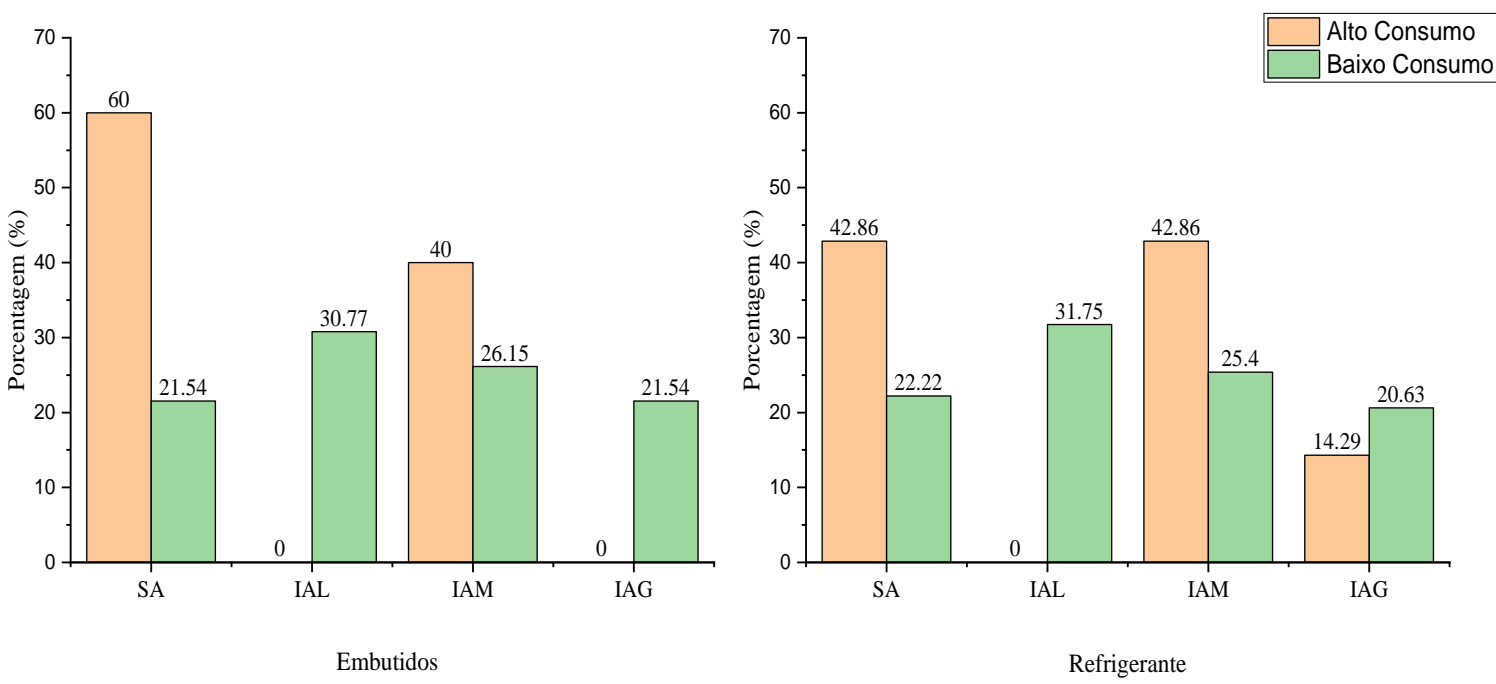

FIGURA A: Porcentagem de consumo para alimentos de baixo valor nutricional em participantes classificados em Segurança Alimentar e Nutricional. Segurança Alimentar (SA), Insegurança Alimentar Leve (IAL), Insegurança Alimentar Moderada (IAM), Insegurança Alimentar Grave (IAG).
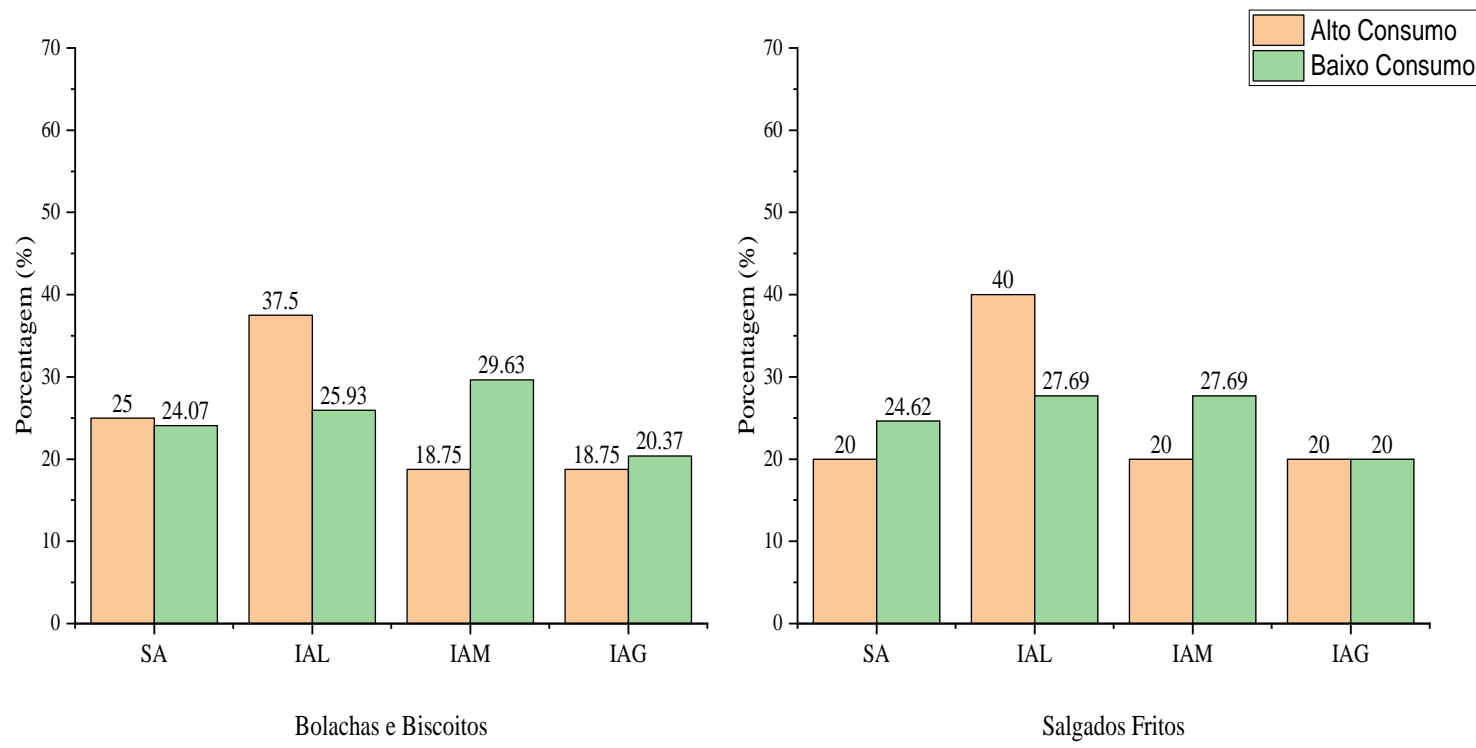

FIGURA B: Porcentagem de consumo para alimentos de baixo valor nutricional em participantes classificados em Insegurança Alimentar Leve. Segurança Alimentar (SA), Insegurança Alimentar Leve (IAL), Insegurança Alimentar Moderada (IAM), Insegurança Alimentar Grave (IAG). 


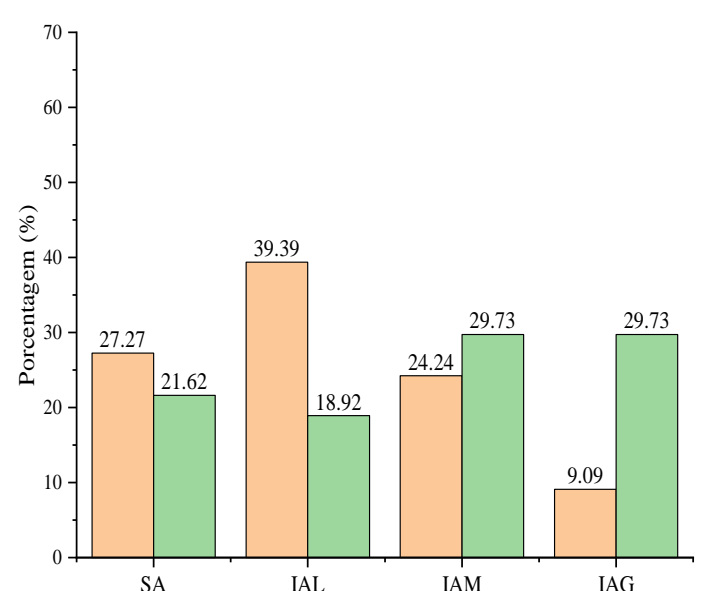

Leite e Iogurte

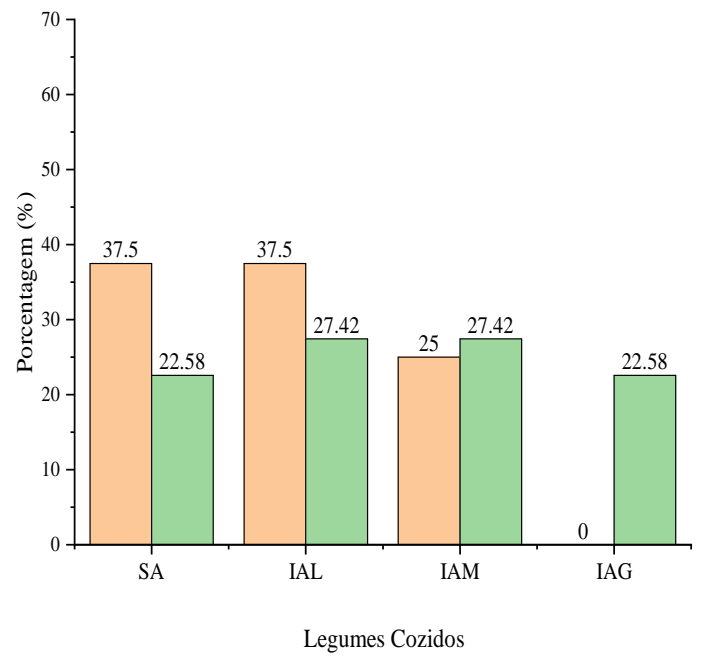

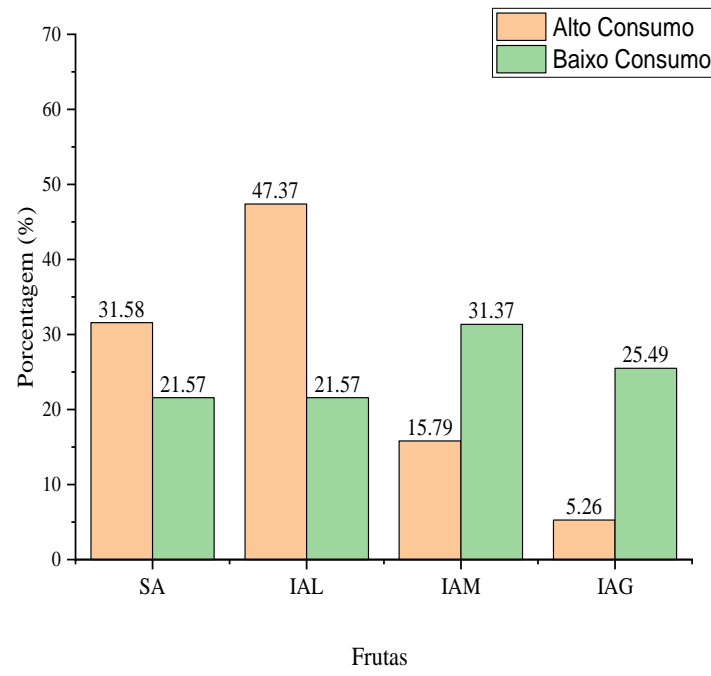

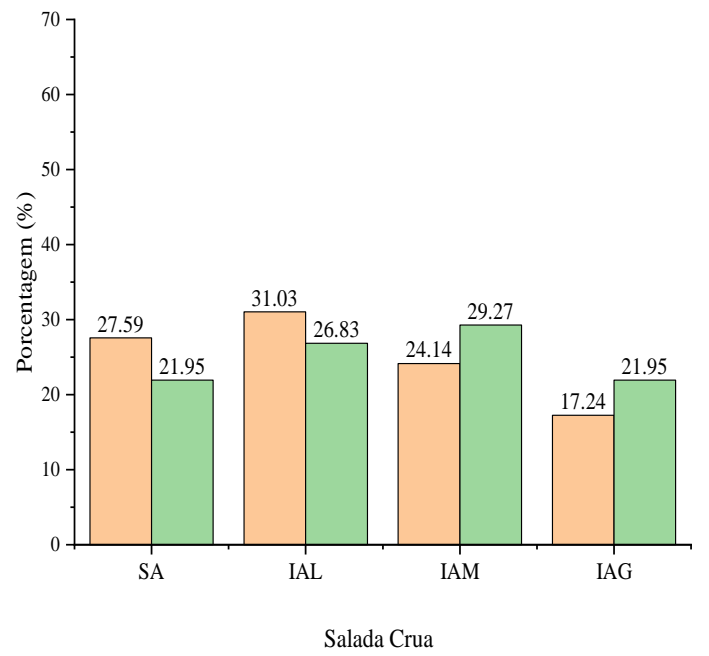

FIGURA C: Porcentagem de consumo para alimentos de alto valor nutricional em participantes classificados em Insegurança Alimentar Leve. Segurança Alimentar (SA), Insegurança Alimentar Leve (IAL), Insegurança Alimentar Moderada (IAM), Insegurança Alimentar Grave (IAG).

TABELA 3: Escolhas obtidas de ambos os grupos pelo Questionário de Frequência Alimentar para alimentos de alto valor nutricional.

\begin{tabular}{llcccc}
\hline Alimento & Condição & Segurança & \multicolumn{3}{c}{ Insegurança alimentar } \\
\cline { 5 - 6 } s & & alimentar & Leve & Moderada & Grave \\
\hline Salada & Alto & $8 / 29(27,59 \%)$ & $9 / 29(31,03 \%)$ & $7 / 29(24,14 \%)$ & $5 / 29(17,24 \%)$ \\
crua & consumo & $9 / 41(21,95 \%)$ & $11 / 41$ & $12 / 41$ & $9 / 29(21,95 \%)$ \\
& Baixo & & $(26,83 \%)$ & $(29,27 \%)$ & \\
Legumes & consumo & & & & \\
cozidos & Alto & $3 / 8(37,5 \%)$ & $3 / 8(37,5 \%)$ & $2 / 8(25 \%)$ & $0 / 8(0 \%)$ \\
& consumo & $14 / 62$ & $17 / 62$ & $17 / 62$ & $14 / 62$ \\
& Baixo & $(22,58 \%)$ & $(27,42 \%)$ & $(27,42 \%)$ & $(22,58 \%)$ \\
Frutas & consumo & & & & \\
& Alto & $6 / 19(31,58 \%)$ & $9 / 19(47,37 \%)$ & $3 / 19(15,79 \%)$ & $1 / 19(5,26 \%)$ \\
& consumo & $11 / 51$ & $11 / 51$ & $16 / 51$ & $13 / 51$ \\
& Baixo & $(21,57 \%)$ & $(21,57 \%)$ & $(31,37 \%)$ & $(25,49 \%)$ \\
Feijão & consumo & & & & \\
& Alto & $14 / 55(25,5 \%)$ & $15 / 55(27,3 \%)$ & $15 / 55(27,3 \%)$ & $11 / 55(20,0 \%)$ \\
& consumo & $3 / 15(20,0 \%)$ & $5 / 15(33,3 \%)$ & $4 / 15(26,7 \%)$ & $3 / 15(20,0 \%)$ \\
& Baixo & & & &
\end{tabular}


consumo

\begin{tabular}{llcccc} 
Leite e & Alto & $9 / 33(27,27 \%)$ & $13 / 33$ & $8 / 33(24,24 \%)$ & $3 / 33(9,09 \%)$ \\
iogurte & consumo & $8 / 37(21,62 \%)$ & $(39,39 \%)$ & $11 / 37$ & $11 / 37$ \\
& Baixo & & $7 / 37(18,92 \%)$ & $(29,73 \%)$ & $(29,73 \%)$ \\
& consumo & & & \\
\hline
\end{tabular}

Embora o grupo classificado em IAL apresente grande consumo de alimentos de alto valor nutricional, é preocupante o consumo de alimentos altamente calóricos. Outro ponto a se destacar, é o fato dos participantes classificados em SAN não apresentarem boa qualidade na alimentação pelo consumo exacerbado de alimentos calóricos (Figura A). Esse dado demonstra que estar em SAN não significa ter uma alimentação de qualidade, mas sim, o acesso a todos os tipos de alimento.

$\mathrm{O}$ PBF foi o resultado da introdução da democracia participativa em que os movimentos de descentralização de políticas públicas foram voltados para o controle social, por meio das execuções de políticas sociais e participação dos brasileiros, gerando-se melhorias na educação, Sistema Único de Saúde (SUS), previdência social, assistência social e direcionada para a população mais suscetível a fome, de forma a enfrentá-la e promover SAN (CARVALHO; SANTOS, 2018).

De acordo com Silvani et al. (2018), o consumo de alimentos pouco nutritivos e altamente energéticos é resultado da transição nutricional que o Brasil vivencia, o padrão alimentar expressava maior tendência para o consumo de produtos in natura como o arroz e o feijão e hoje tende para alimentos calóricos e pouco nutritivos. Segundo Schimidt et al. (2011), as Doenças Crônicas Não Transmissíveis (DCNTs) expõe um grave problema de saúde pública, predominando em famílias de baixa renda e que estas doenças estão progredindo cada vez mais pelo excesso de peso oriundas de mudanças desfavoráveis na dieta habitual.

Morales e Berkowitz (2016) apontam em um estudo que a insegurança alimentar engloba uma série de situações, desde a fome até a preocupação de que a comida acabe. Com isso, ocorre uma menor ingesta calórica e consequentemente, redução da ingesta de nutrientes, tendendo a consumir dietas com menor qualidade nutricional.

Um estudo de Silvani et al. (2018), envolvendo beneficiários do BF e usuários da Estratégia de Saúde da Família (ESF), evidenciou hábitos alimentares não saudáveis para ambos os grupos e ainda reforça que apesar dos benefícios oferecidos pelos programas de transferência de renda, é necessário que existam ações de educação e avaliação nutricional a fim de melhorar os resultados.

Outro estudo realizado no município Vale do Sol (RS) por Witt et al. (2017), os autores avaliaram a frequência de IA de 59 famílias beneficiárias do PBF associando com grau de escolaridade do responsável familiar e os resultados obtidos para SA, IAL, IAM e IAG foram respectivamente 22,0\%, 78,0\%, 89,1\%, 10,9\% sendo evidente o número alarmante de pessoas em situação de insegurança alimentar leve e moderada.

Favaro et al. (2007) em estudo realizado no ano de 2004 no Mato Grosso do Sul, com população indígena beneficiários do PBF, dentre outros programas de transferência de renda, tiveram como resultado prevalência de $22,4 \%, 32,7 \%$, $20,4 \%$, respectivamente para insegurança leve, moderada e grave, observando que somente $24,5 \%$ das famílias vivenciavam a segurança alimentar.

Suzart e Ferreira (2018), desenvolveram um estudo transversal analítico em 2015 na cidade de Vitória da Conquista- BA, com famílias beneficiárias do PBF 
utilizando também a EBIA, e, de acordo com os resultados, 40,8\% da amostra estava em situação de IA leve, 26,9 \% moderada e IA grave/severa 5,7\%.

\section{CONCLUSÃO}

Nas condições em que o estudo foi realizado, e avaliando os resultados dos questionários, observou-se que a situação de SAN ou IA de um município reflete as condições nutricionais, pela qualidade da alimentação como também, condições socioeconômicas, pelo acesso aos alimentos. Ressalta-se que, mesmo com o aporte do PBF, constatou-se que não se atingiu os objetivos propostos pelo PBF quanto a SAN dos participantes do estudo, pela elevada quantidade de alimentos pouco nutritivos consumidos tanto pelos beneficiários, quanto pelos não beneficiários, mostrando que, o acesso ao alimento promovido pelo programa não é acompanhado pela qualidade na alimentação.

\section{REFERÊNCIAS}

BRASIL. Ministério da Saúde (MS). Protocolo do Sistema de Vigilância Alimentar e Nutricional - SISVAN na assistência à saúde. Brasília: MS; 2008. Disponível em: < http://189.28.128.100/nutricao/docs/geral/protocolo_sisvan.pdf> Acesso em: 4 de outubro de 2018.

CARVALHO, M. G.; SANTOS, L. L. P. O Controle social na efetivação das políticas públicas com ênfase na assistência social. Revista De Psicologia, v. 12, n. 41, p. 760-773, 2018.

COELHO, P.; MELO, A. S. S.A. Impacto do Programa "Bolsa Família" sobre a qualidade da dieta das famílias de Pernambuco no Brasil. Revista Ciência \& Saúde Coletiva, Pernambuco, v. 22, n. 2, 393-401, julho. 2017.

FARIA, A. A.; DIAS, R. B. Direito à alimentação, transferência de renda e progressividade: o caso do programa bolsa família no Brasil. Revista Jurídica da Presidência, São Paulo, v. 18, n. 114, p. 145-168, maio. 2016.

FÁVARO, T.; RIBAS, D. L. B.; ZORZATTO, J. R.; SEGALL-CORREAA, A. M.; PANIGASSI, G. Segurança alimentar em famílias indígenas Teréna, Mato Grosso do Sul, Brasil. Cadernos de Saúde Pública, v. 23, p. 785-793, 2007.

FERREIRA. H. S.; SOUZA, M. E. C. A.; MOURA, F. A.; HORTA, B. L. Prevalência e fatores associados à Insegurança Alimentar e Nutricional em famílias dos municípios do norte de Alagoas, Brasil. Revista Ciência \& Saúde Coletiva. Alagoas, v. 19, n. 5, maio/jun. 2014.

MORALES, M. E.; BERKOWITZ, S. A. The relationship between food insecurity, dietary patterns, and obesity. Current nutrition reports, v. 5, n. 1, p. 54-60, 2016.

PINTO, C. A.; MORAIS, D. C.; SILVA, L. A.; FRANCESCHINI, S. C. C.; PRIORE, S. I. Insegurança alimentar e estado nutricional de famílias beneficiárias do Programa Bolsa Família. Journal of Management \& Primary Health Care, Pernambuco, v. 7, n. 1 , p. 46-46, jul/2017. 
SEGALL-CORRÊA, A.; ESCAMILLA, R.P.; SAMPAIO, MF.F.A; MARIN-LEON, L.; PANIGASSI, G. M. et al. Acompanhamento e avaliação da segurança alimentar de famílias brasileiras: validação de metodologia e de instrumento de coleta de informação (Urbano). Campinas: Universidade Estadual de Campinas; 2003.

SCHMIDT, M. I. DUNCAN, B. B.; SILVA, G. A.; MENEZES, A. M.; MONTEIRO, C. A.; et al. Chronic non-communicable diseases in Brazil: burden and current challenges. The Lancet, Porto Alegre: v. 377, n. 9781, p. 1949-1961, 2011.

SILVA, P. D. N.; KERNKAMP, C. L.; BENNEMANN, R. M. Insegurança alimentar: as desigualdades de renda e a vulnerabilidade social como resultados na alimentação dos idosos. Revista Uningá Review, Maringá, v. 16, n. 2, 2018.

SUZART, A. S.; FERREIRA, A. P. Evaluation of the Bolsa Família Program (PBF) on food and nutritional security in Beneficiary families, Vitória da Conquista: v. 19 , n. 3, p. 585-595, 2018.

SILVANI, J.; BUSS, C.; PENA, G. G.; RECCHI, A. F.; WENDLAND, E. M. Consumo alimentar de usuários do Sistema Único de Saúde segundo o tipo de assistência e participação no Bolsa Família. Ciência \& Saúde Coletiva, Porto Alegre: v. 23, p. 2599-2608, 2018.

VASCONCELLOS, A. B. P. A.; MOURA, L. B. A. Segurança alimentar e nutricional: uma análise da situação da descentralização de sua política pública nacional. Cadernos de Saúde Pública, Brasília: v. 34, p. 13, 2018.

YUYAMA, L. K. O.; DANIEL, V. P.; ISHIKAWA, N. K.; MEDEIROS, J. F.; KEPPLE, A. W.; et at. Percepção e compreensão dos conceitos contidos na Escala Brasileira de Insegurança Alimentar, em comunidades indígenas no estado do Amazonas, Brasil. Revista de Nutrição, Campinas, v. 21(supl.), p. 53s-63s, jul./ago. 2008.

WITT, F.; ETGES, B. I.; LEPPER, L. Insegurança alimentar em famílias do programa Bolsa Família. Segurança Alimentar e Nutricional, v. 25, n. 2, p. 85-93, 2017. 